\title{
EXTENSION PROBLEM TO AN INVERTIBLE MATRIX
}

\author{
VADIM TOLOKONNIKOV
}

(Communicated by Theodore W. Gamelin)

\begin{abstract}
The extension problem for rectangular matrices with values in Banach algebra to an invertible square matrix is investigated. For this problem to be solvable for a matrix $D$, the following condition is necessary: for every maximal ideal $m$ of the algebra, the numerical matrix $D(m)$ must have maximal rank. This condition is sufficient for many algebras, for example, for the algebras $H^{\infty}(R)$ of bounded analytic functions in a plane finitely connected domain $R$ and to Sarason subalgebras in the algebra $H^{\infty}$.
\end{abstract}

For any algebra $A$ let us introduce spaces of vectors and matrices with values in $A$ :

$$
A_{n}:=\left\{a_{i} \in A: 1 \leq i \leq n\right\}, \quad A_{n, k}:=\left\{a_{i, j} \in A: 1 \leq i \leq n, 1 \leq j \leq k\right\} .
$$

All algebras will be assumed to be commutative, Banach, and to have unit (briefly $\mathrm{CBU}$ ) over the field $\mathbb{C}$ unless otherwise declared. When $k<n$ there exists a natural projection pr from $A_{n, n}$ on $A_{n, k}$, if $B \in A_{n, n}$ then $\operatorname{pr}(B)_{i, j}=$ $B_{i, j}, 1 \leq i \leq n, 1 \leq j \leq k$.

Let us study the following problem: for which matrices $D$ from $A_{n, k}$ does there exist an invertible matrix $B$ in the algebra $A_{n, n}$ that extends the matrix $D, D=\operatorname{pr}(B)$ ? For the solvability of this problem the following local condition is necessary: for every maximal ideal $m$ of the algebra $A$ the numerical matrix $D(m)$ from $\mathbb{C}_{n, k}$ must have maximal rank. This is equivalent to the next condition: for every maximal ideal $m$ of the algebra, the numerical matrix $D(m)$ must be left invertible,

$$
\inf \left\{|D(m) y|: m \in \mathfrak{M}(A), y \in \mathbb{C}_{k},|y|=1\right\}>0 .
$$

Let us say that for the algebra $A$ condition (C) is fulfilled if for all $k<n$ and all matrix-valued functions $D$ with condition (LI) there exists some extension to an invertible element of the algebra $A_{n, n}$

$$
\forall n \forall k \forall D \in A_{n, k} D \in(\mathrm{LI}) \Rightarrow \exists B \in A_{n, n}, \quad D=\operatorname{pr}(B), B^{-1} \in A_{n, n} .
$$

Such algebras were studied in [5], where it was shown that for all algebras $A$ with maximal ideal space $\mathfrak{M}(A)$ homotopic to a point or CW-complex of

Received by the editors April 22, 1991 and, in revised form, July 17, 1991.

1991 Mathematics Subject Classification. Primary 30H05, 46J15; Secondary 13C10.

Key words and phrases. Banach algebras, subalgebras of $H^{\infty}$, matrices, projective modules, vector bundles. 
dimension one or two, condition (C) is fulfilled. In [10, Theorem 4] the author proved, with the help of the Lax-Halmos theorem (briefly LH-theorem), that the algebra $H^{\infty}$ of bounded analytic functions in the unit disc $\mathbb{D}=\{z \in \mathbf{C}:|z|<1\}$ satisfies the same condition. The connection between condition (C) and the LHtheorem is discussed in the first theorem of this paper, where it is shown that condition $(\mathrm{C})$ is equivalent to some weak variant of the LH-theorem, given later. Examples of subalgebras in $H^{\infty}$ with this condition (Sarason algebras) are given. For algebras of bounded analytic functions in plane finitely connected domains, this condition is fulfilled too. Theorem 4, in the second part of the paper, as well as its first corollary were mainly proved in [5], and we present them here for completeness. In the last theorem of this paper the connection between the dimension of the maximal ideal space of an algebra and property (C) is investigated. For example, it is proved there that if the maximal ideal space of an algebra is a CW-complex of dimension not greater than four, then condition $(\mathrm{C})$ is fulfilled, but for dimension five this is not always true.

\section{Condition (wLH) AND SUbalgebras of $H^{\infty}$}

Let us associate with every matrix $D$ from $A_{n, k}$ the transposed matrix $D^{\mathrm{T}}$ from $A_{k, n}$ and the operator $M_{D, A}$ of multiplication by the matrix $D$, acting from $A_{k}$ to $A_{n}$

$$
\begin{gathered}
D_{i, j}^{\mathrm{T}}:=D_{j, i}, \quad 1 \leq i \leq k, 1 \leq j \leq n ; \\
M_{D, A}(C):=D \cdot C, \quad C \in A_{k} .
\end{gathered}
$$

Let us say that a CBU-algebra $A$ satisfies condition (wLH) if for every matrix $G$ from $A_{k, n}$ with the property $G^{\mathrm{T}} \in(\mathrm{LI})$ there exists a matrix $H$ from $A_{n, n-k}$ such that for the kernel of the multiplication operator the following formula is true:

$$
\operatorname{ker}\left(M_{G, A}\right)=\operatorname{Im}\left(M_{H, A}\right)=H \cdot A_{n-k} .
$$

Theorem 1. For every $C B U$-algebra, conditions $(\mathrm{wLH})$ and $(\mathrm{C})$ are equivalent.

The following lemma was proved in [1] with the help of a noncommutative generalization of the Gelfand theory of the maximal ideal for commutative Banach algebras. We present here another proof.

Lemma 1. For every $C B U$-algebra $A$ and for every matrix $D$ from $A_{n, k}$ with property $D \in(\mathrm{LI})$ one can find a matrix $G$ from $A_{k, n}$ such that $G \cdot D=I_{k}$, where $I_{k}$ is the identity $k$-dimensional matrix.

Proof of Lemma 1. If $\alpha$ is a $k$-element subset in the index interval from 1 to $n$, let $L_{\alpha}$ be the determinant of submatrix of the matrix $D$ with numbers of columns from $\alpha$. Then $L$ is in $A_{m}$ for some number $m$. Since the matrix $D$ has maximal rank elsewhere on $\mathscr{M}(A)$, the vector $L$ is nonzero on $\mathscr{M}(A)$ and there exists a vector $N \in A_{m}$ such that $\sum_{\alpha} L_{\alpha} \cdot N_{\alpha}=1$. Let $L_{\alpha}^{i j}$ be the algebraic supplement of the element $D_{j i}$ in $L_{\alpha}$ if $j \in \alpha$ and zero otherwise, and let $G_{i j}:=\sum_{\alpha} L_{\alpha}^{i j} \cdot N_{\alpha}$. According to the well-known properties of determinant, $\sum_{j} L_{\alpha}^{i j} \cdot D_{j k}=\delta_{i k} \cdot L_{\alpha}$ and so,

$$
(G D)_{i k}=\sum_{j} G_{i j} \cdot D_{j k}=\sum_{j, \alpha} L_{\alpha}^{i j} \cdot N_{\alpha} \cdot D_{j k}=\delta_{i k} \cdot\left(\sum_{\alpha} L_{\alpha} \cdot N_{\alpha \alpha}\right)=\delta_{i k} .
$$


Proof of Theorem 1. Let the algebra $A$ satisfy condition (wLH) and let $D \in$ $A_{n, k}, D \in(\mathrm{LI})$. Then according to Lemma 1 one can find a matrix $G$ from $A_{k, n}$ such that $G \cdot D=I_{k}$. Let us apply the property (wLH) to the matrix $G$; then we have $\operatorname{ker}\left(M_{G, A}\right)=H \cdot A_{n-k}$ for some matrix $H$ from $A_{n, n-k}$. For the matrix $J:=I_{n}-D \cdot G$, we have $G \cdot J=0$. Let us define $J_{i}$ as the $i$ th column of the matrix $J$; then $J_{i} \in A_{n}, G \cdot J_{i}=0, J_{i} \in \operatorname{Ker}\left(M_{G}\right)=H \cdot A_{n-k}$, and for some column $S_{i}$ from $A_{n-k}$ we have $J_{i}=H \cdot S_{i}$. Let us define the matrix $S$ as $S=\left(S_{1}, S_{2}, \ldots, S_{n}\right) \in A_{n-k, n}$; then $J=H \cdot S$ and $D \cdot G+H \cdot S=I_{n}$. So $D=\operatorname{pr}(F)$, where the matrix $F:=(D, H)$ is invertible in $A_{n, n}$ and $F^{-1}=\left(\begin{array}{l}G \\ S\end{array}\right)$.

On the other hand, if $G$ lies in $A_{k, n}$ and $G^{\mathrm{T}}$ satisfies condition (LI), then it follows from condition (C) that there exists a matrix $L$ invertible in $A_{n, n}$ such that $G^{\mathrm{T}}=\operatorname{pr}(L)$. Let $L^{\mathrm{T}}=\left(\begin{array}{c}G \\ S\end{array}\right)$, where $S \in A_{n-k, n}$ and $M:=\left(L^{\mathrm{T}}\right)^{-1}$, $M=(D, H), D \in A_{n, k}, H \in A_{n, n-k}$. Then $M \cdot L^{\mathrm{T}}=D \cdot G+H \cdot S=I_{n}$ and, therefore, $\operatorname{ker}\left(M_{G, A}\right) \subset \operatorname{Im}\left(M_{H, A}\right)$. Because $M$ is both right and left inverse to $L^{\mathrm{T}}$, we have $L^{\mathrm{T}} \cdot M=I_{n}$ and $G \cdot H=0$; therefore, $\operatorname{Im}\left(M_{H, A}\right)=$ $\operatorname{ker}\left(M_{G, A}\right)$.

Let us introduce some new notions for formulating the LH-theorem for the algebra $H^{\infty}$. A linear space $M$ is called a module over an algebra $A$ if there exists an $M$-valued bilinear form on $A \times M$. An $A$-invariant subspace of any module is called a submodule.

LH-Theorem. If $M$ is a weak-star closed submodule of the $H^{\infty}$-module $H_{n}^{\infty}$, then for some $k$ the module $M$ can be represented as $M=\Psi \cdot H_{k}^{\infty}$, where $\Psi \in H_{n, k}^{\infty}$ is a left unimodular matrix, that is, $\Psi^{*} \cdot \Psi=I_{k}$. If two such modules $\Psi \cdot H_{k}^{\infty}$ and $\boldsymbol{\Theta} \cdot H_{m}^{\infty}$ are equal, then $k=m$ and $\Psi=\boldsymbol{\theta} \cdot V$, where $V$ is $a$ matrix from the group $U(k)$ of unitary operators in $\mathbb{C}^{k}$.

For example this theorem can be taken from monograph [7], where in Corollary 6 on p. 33 the description of a closed submodule of the $H^{\infty}$-module $H_{n}^{2}$ was given in detail and on pp. 45-47 necessary modifications for the case of other spaces is described.

A subalgebra $X$ of the algebra $L^{\infty}(\mathbb{T})$ of bounded functions on the circle $\mathbb{T}=\{z \in \mathbb{C}:|z|=1\}$ containing $H^{\infty}$ is called $a$ Douglas algebra if it is closed, and a generalized Douglas algebra if

$$
f \in X,\|f\|_{\infty}<1 \Rightarrow 1 /(1-f) \in X
$$

Let us define for every such algebra (briefly GD-algebra) the analytic Sarason algebra $Q X A:=X \cap H^{\infty}$. In the author's papers [11,12], the Corona theorem for such algebras is proved, so the disk $\mathbb{D}$ is dense in $\mathfrak{M}(Q X A)$ and condition (LI) can be rewritten as

$$
\inf \left\{|D(z) y|: z \in \mathbb{D}, y \in \mathbb{C}_{k},|y|=1\right\}>0 .
$$

Theorem 2. For every GD-algebra $X$ the Sarason algebra $Q X A$ fulfills condition (C).

Proof. Let us check that the algebra $Q X A$ fulfills condition (wLH). Let a matrix $G$ belong to $Q X A_{k, n}$ and suppose that for $G^{\mathrm{T}}$ condition (LI) is fulfilled. Then according to Lemma 1 there exists $D$ from $Q X A_{n, k}$ such that $G \cdot D=I_{k}$. 
Since $G$ lies in $H_{k, n}^{\infty}, \operatorname{ker}\left(M_{G, H^{\infty}}\right)$ is a submodule of the $H^{\infty}$-module $H_{n}^{\infty}$ that is closed in weak-star topology. According to the LH-theorem, $\operatorname{ker}\left(M_{G, H^{\infty}}\right)$ is equal to $H \cdot H_{n-k}^{\infty}$ for a left unimodular matrix $H$ from $H_{n, n-k}^{\infty}$. If we repeat the proof of the previous theorem, there exists a matrix $S$ from $H_{n-k, n}^{\infty}$ such that $D \cdot G+H \cdot S=I_{n}, D=\operatorname{pr}(F)$, where $F:=(D, H)$ and $F^{-1}=\left(\begin{array}{l}G \\ S\end{array}\right)$. Let us check that $S \in Q X A_{n-k, n}$,

$$
\begin{gathered}
S=H^{*} H S=H^{*}\left(I_{n}-D \cdot G\right) \in \overline{H_{n-k, n}^{\infty}} \cdot Q X A_{n, n} \subset \overline{X_{n-k, n}}, \\
S \in H_{n-k, n}^{\infty} \cap \overline{X_{n-k, n}}=Q X A_{n-k, n} .
\end{gathered}
$$

That is why $F^{-1} \in Q X A_{n, n}$, and so $\operatorname{det}(F) \in Q X A$, $\operatorname{det}(F)^{-1} \in H^{\infty}$. As a special case of the Corona theorem proved in [11, 12], we have for such algebras $\operatorname{det}(F)^{-1} \in Q X A$. According to the formula for an inverse matrix we have $F \in Q X A_{n, n}$ and $H=\operatorname{pr}(F) \in Q X A_{n, n-k}$. To complete the proof let us check that if $g \in \operatorname{ker}\left(M_{G, Q X A}\right)$ then $g \in H \cdot Q X A_{n-k} . \operatorname{But} \operatorname{ker}\left(M_{G, Q X A}\right) \subseteq$ $\operatorname{ker}\left(M_{G, H^{\infty}}\right)=H \cdot H_{n-k}^{\infty}$, and so $g=H \cdot v, v \in H_{n-k}^{\infty}$; then $v=H^{*} \cdot g \in$ $\left(\overline{H_{n-k, n}^{\infty}} \cdot \overline{X_{n}}\right) \subseteq \overline{X_{n-k}}$ and $v \in Q X A_{n-k}$.

In particular, Theorem 2 can be applied to the usual Sarason algebras for the case when $X$ is a Douglas algebra. Another example: let $B_{p q A}^{s}$ be the analytic Besov space of functions in the disk $\mathbb{D}, 1 \leq p, q \leq \infty, s>0$; then $H^{\infty} \cap B_{p q A}^{s}$ and $\operatorname{Mult}\left(B_{p q A}^{s}\right)$ are $Q X A$-algebras for some GD-algebras $X$ [11, 12], where $\operatorname{Mult}\left(B_{p q A}^{s}\right)$ is the algebra of multipliers of the space $B_{p q A}^{s}$.

Previously for the Banach algebras, the property of coherence was examined, which can be formulated in the following way: for every matrix function $Q$ from $A_{n, k}$, the module $\operatorname{ker}\left(M_{G, A}\right)$ is finitely generated. This property is considerably different from property (wLH) defined in this paper, which can be seen from the result: the Sarason algebra $Q X A$ corresponding to a Douglas algebra $X$ is coherent if and only if $Q X A=H^{\infty}$ [6].

Theorem 3. For any finitely connected plane domain $R$ the algebra $H^{\infty}(R)$ of bounded analytic functions fulfills condition (C).

The proof can be obtained from Theorem 1 and

Lemma 2. If $M$ is a weak-star closed submodule of the $H^{\infty}(R)$-module $H^{\infty}(R)_{n}$, then for some $k$ the module $M$ can be represented as $M=H$. $H^{\infty}(R)_{k}$, where $H$ from $H^{\infty}(R)_{n, k}$ is a left invertible matrix.

Proof of the lemma. The domain $R$ has $\mathbb{D}$, the unit disk, as its universal covering domain; let $G$ be the associated group of automorphisms of $\mathbb{D}$. The algebra $H^{\infty}(R)$ can then be identified with the subalgebra $H_{G}^{\infty}$ of $G$-invariant functions from the algebra $H^{\infty}$, and the module $M$ can be identified with a $H_{G}^{\infty}$-submodule in $H_{G, n}^{\infty}$. Let $N$ be the weak-star closed $H^{\infty}$-module generated by $M$. Then by the LH-theorem, $N=\Psi \cdot H_{k}^{\infty}$, where $\Psi$ is a left unimodular function. If $A$ is an element of the group $G$ then $\Psi \cdot H_{k}^{\infty}=\Psi \circ A \cdot H_{k}^{\infty}$, and so $\Psi(A(z))=\Psi(z) \cdot \alpha(A)$ for all $z$ in $\mathbb{D}$, where $\alpha(A)$ is a matrix from $U(k)$. It is evident that such a mapping $\alpha$ is a homomorphism of the group $G$ to the group $U(k)$. Let $H^{\infty}(\mathbb{D}, \alpha)$ be the space of all functions in $H_{k}^{\infty}$ with property $f(A(z))=\alpha(A)^{*} \cdot f(z)$ for all $z$ from $\mathbb{D}$ and all $A$ from $G$. Then 
$N \cap H_{G}^{\infty}=\Psi \cdot H^{\infty}(\mathbb{D}, \alpha)$, and by a theorem of Grauert [3] there exists an invertible function $\Omega$ in $H_{k, k}^{\infty}$ with the property that $\Omega(A(z))=\alpha(A)^{*} \cdot \Omega(z)$ for all $z$ in $\mathbb{D}$ and all $A$ in $G$. Then $\Psi \cdot \Omega \in H_{G, n, k}^{\infty}$ and $N \cap H_{G}^{\infty}=\Psi \cdot \Omega \cdot H_{G, k}^{\infty}$ and the function $\Psi \cdot \Omega$ corresponds to some left invertible function $H$ from $H^{\infty}(R)_{n, k}$. The only fact we have to check is the equality $N \cap H_{G}^{\infty}=M$. By Forelli's theorem [2] there exists a weak-star continuous $H_{G}^{\infty}$-linear projection $F$ from $H^{\infty}$ to $H_{G}^{\infty}$. If $f \in N \cap H_{G}^{\infty}$ then $f$ is the limit of a sequence of functions $f_{k}$ of the form $f_{k}=\sum_{i=1}^{n_{k}} g_{i k} \cdot h_{i k}$ where $g_{i k} \in H^{\infty}, h_{i k} \in M$. Since $F\left(g_{i k} \cdot h_{i k}\right)=F\left(g_{i k}\right) \cdot h_{i k}$, we have $F\left(f_{k}\right) \in M$ and then $f \in M$.

\section{CONDITION (C) IN TERMS OF MODULES AND VECTOR BUNDLES}

Condition (C) can be reformulated in terms of modules and vector bundle theory. We recall some definitions given in more detail in [9] and [4], respectively. A module $M$ over an algebra $A$ is called free if for some $k$ it is isomorphic to the module $A_{k}$ and projective if for some module $N$ the direct sum of modules $M \oplus N$ is free. Two modules $M$ and $N$ are called stably isomorphic if for some $k$ the modules $M \oplus A_{k}$ and $N \oplus A_{k}$ are isomorphic.

A complex vector bundle $\xi$ over a compact $K$ is a continuous function from the topological space $E$ to $K$ such that for all $x$ in $K$ the set $p^{-1}(x)$ has the structure of the vector space $\mathbb{C}^{k}$ and one can find a neighborhood $U$ of the point $x$, for which the subbundle $p^{-1}(U) \rightarrow U$ is trivial, where a bundle is trivial if it is isomorphic to the canonical projection from $U \times \mathbb{C}^{k}$ to the first factor of the cartesian product. A section of a bundle $\xi$ is a continuous function $q$ from $K$ to $E$ such that $p \circ q=\mathrm{id}_{K}$. By the symbol $\Omega^{n}$ we mean the $n$-dimensional trivial bundle over $K$, isomorphic to the projection from $K \times \mathbb{C}^{k}$ into $K$. Two bundles $\xi$ and $\eta$ are called stationarily equivalent if for some $m$ and $n$ the direct sums $\xi \oplus \Omega^{m}$ and $\eta \oplus \Omega^{n}$ are isomorphic. Real vector bundles can be defined in a completely similar way.

Theorem 4. Let $A$ be a $C B U$-algebra with maximal ideal space $K$. The following conditions are equivalent:

(1) The algebra $A$ satisfies condition (C).

(2) The algebra $C(K)$ of all continuous functions on the compact $K$ satisfies condition $(\mathrm{C})$.

(3) Every finitely generated projective module over the algebra $A$ that is stably isomorphic to a free one is free.

(4) The same as (3) but for the algebra $C(K)$.

(5) Every vector bundle over $K$ that is stationarily equivalent to the trivial one is trivial.

Proof. The equivalence $(1) \Leftrightarrow(2)$ is a consequence of the following theorem in [5]: a matrix $C$ from $A_{n, k}$ can be extended to an invertible matrix $B$ from $A_{n, n}$ if and only if it can be extended to an invertible matrix $D$ in $C(K)_{n, n}$. The equivalence $(3) \Leftrightarrow(4)$ is a consequence of the theorem in [8] about the existence of natural isomorphism between categories of finitely generated projective modules over the algebras $A$ and $C(K)$. Both these theorems can be found in $[9, \mathrm{pp} .179,196]$. The equivalence $(4) \Leftrightarrow(5)$ is a consequence of the isomorphicity of the category of the vector bundles and the category of finitely 
generated projective modules over the algebra $C(K)$. The isomorphism associates with every vector bundle over $K$ the space of its continuous sections, and it is easy to verify that this will be a projective $C(K)$ module.

Let us check that $(5) \Rightarrow(2)$. Let condition (5) of the theorem be true and $D \in(\mathbf{L I})$. Then the matrix $D$ from $C(K)_{n, k}$ determines some $k$-dimensional subbundle $E$ of the trivial $n$-dimensional bundle $\Omega^{n}$. $E$ itself is trivial too: $E$ is isomorphic to $\Omega^{k}$. Then for the orthogonal bundle $E^{\perp}$ we have $E^{\perp} \oplus \Omega^{k} \cong$ $\Omega^{n}$, and $E^{\perp}$ is stationarily equivalent to the trivial bundle $\Omega^{n-k}$. By our assumption, $E^{\perp}$ is equivalent to the trivial bundle and can be defined by an element $F$ in $C(K)_{n, n-k}$. Then the matrix $G:=(D, F)$ is an invertible element of $C(K)_{n, n}$ and $D=\operatorname{pr}(G)$.

Let us check that $(2) \Rightarrow(5)$. Suppose condition (2) of the theorem is true and the bundle $E$ is stationarily equivalent to the trivial bundle $\Omega^{m}$, so that there exists a number $k$ such that $E \oplus \Omega^{k}$ is equivalent to $\Omega^{m+k}$. Then the subbundle $\Omega^{k}$ can be defined by some matrix $C \in C(K)_{m+k, k}$. Since the inclusion $E$ in $\Omega^{m+k}$ is an injection, condition (LI) is satisfied for the matrix $C$ and we can apply the theorem to it. So for some trivial bundle $F$ we have that the bundles $F \oplus \Omega^{k}$ and $\Omega^{m+k}$ are isomorphic, so $F$ is isomorphic to $\Omega^{m}$. Now it is easy to verify that the orthogonal projection $F$ on $E$ in $\Omega^{k+m}$ defines an isomorphism of $E$ to the trivial bundle.

Let us say that for a compact $K$ the condition (Ext) is satisfied if for the algebra $C(K)$ all conditions of Theorem 4 are met. Because the homotopy between the topological spaces defines an isomorphism between the vector bundles, we have following consequences:

Corollary 1 (Lin [5]). Let $K_{1}, K_{2}$ be two homotopy compacts and suppose condition (Ext) is satisfied for $K_{1}$. Then condition (Ext) is satisfied for $K_{2}$ as well; condition (Ext) is fulfilled for $K$ if the compact $K$ is homotopic to a point.

Corollary 2. Condition (Ext) is satisfied for the compact $\mathfrak{M}(Q X A)$ for all Banach GD-algebras $X$.

It was shown in [5] in our notation that if $K$ is a CW-complex of dimension not greater than two then condition (Ext) is satisfied. In the following theorem we show that the maximal possible dimension for this case is equal to four. In the same theorem, real Banach algebras are investigated, too. The previous discussion does not depend on the choice of the field, but we must change condition (Ext).

Theorem 5. Let the maximal ideal space $\mathfrak{M}(A)$ of some CBU-algebra $A$ be a $C W$-complex of dimension $n$. Then

1. If $A$ is an algebra over the field $\mathbb{C}$ and $n \leq 4$, then the algebra $A$ satisfies condition $(\mathrm{C})$. This statement is not correct for $n=5$ : the algebra $C\left(S^{5}\right)$ does not meet condition $(\mathrm{C})$, where $S^{5}$ denotes the unit sphere of the dimension 5 .

2. If $A$ is an algebra over field $\mathbb{R}$ and $n \leq 1$, then the algebra $A$ satisfies condition (C). This statement is not correct for $n=2$ : the algebra $C_{R}\left(S^{2}\right)$ does not meet condition (C) where $C_{R}\left(S^{2}\right)$ is the algebra of continuous real-valued functions on $S^{2}$.

Lemma 3. (1) Let $K$ be a $C W$-complex of dimension $n$ and $\xi$ be a vector bundle over $K$ of dimension $m$, which is stationarily equivalent to the trivial 
one. If the bundle is over the field $\mathbb{C}$ and $2 m \geq n$, or over the field $\mathbb{R}$ and $m \geq n+1$, then the bundle $\xi$ is trivial.

(2) If the matrix $D$ is from $C(K)_{p, p-1}$ for some compact $K$ and number $p$ and meets condition (LI), then there exists an extension of the matrix $D$ to an invertible one.

Proof of the lemma. The first part of the lemma is proved in [4, Theorem 8.1.5], so let us check the second part. Let $F_{i}$ be the determinant of the matrix obtained by crossing out the $i$ th column in matrix $D, i=1,2, \ldots, p$. Since the rank of the matrix $D$ is $p-1$ everywhere on the compact $K$, the vector $F$ is never zero on the $K$ and there exists a vector $G$ in $C(K)_{p}$, so that

$$
F_{1} G_{1}-F_{2} G_{2}+\cdots+(-1)^{p-1} F_{p} G_{p}=1 .
$$

Now we can take the direct sum $D \oplus G$ as the desired invertible extension.

Proof of the theorem. While proving Theorem 4 we showed that the statement about the triviality of all vector bundles of dimension $m$ over compact $K$ is equivalent to the possibility of finding invertible extensions for all matrices with codimension $m$. The second statement of the lemma guarantees that this problem can always be solved when $m=1$. The affirmative part of the theorem can be obtained from the first part of Lemma 3 with $m=2$.

Some results from [4] are necessary for constructing examples. Let us cut the sphere $S^{n}$ along the subsphere $S^{n-1}$ into two bowls, which topologically are balls $D^{n}$ of the dimension $n$. Then the class $\operatorname{Vect}_{k}\left(S^{n}\right)$ of the isomorphic $k$-dimensional vector bundles on $S^{n}$ can be identified with the $(n-1)$ th homotopy group of the unitary group $U(k): \operatorname{Vect}_{k}\left(S^{n}\right) \cong \pi_{n-1}(U(k))$. Then the class $K\left(S^{n}\right)$ of the stationarily equivalent vector bundles on the sphere $S^{n}$ is isomorphic to $\lim _{k \rightarrow \infty}\left(\operatorname{Vect}_{k}\left(S^{n}\right)\right)$ [4]. Since $\pi_{4}(U(2)) \cong \pi_{4}\left(S^{3}\right) \cong \mathbb{Z}_{2}$ and $\pi_{4}(U(k)) \cong 0$ for $k \geq 3[4]$, we have $\mathbb{Z}_{2} \cong \operatorname{Vect}_{2}\left(S^{5}\right) \neq K\left(S^{5}\right) \cong 0$, so that condition (Ext) is not fulfilled for the sphere $S^{5}$.

Similar calculations can be made for the field $\mathbb{R}$, but in this case we can present a concrete counterexample: the tangential bundle $\tau\left(S^{2}\right)$ of the sphere $S^{2}$ is stationarily equivalent to the trivial bundle, but is not trivial. In terms of matrices: the 3-dimensional field $(x, y, z)$ on the sphere $x^{2}+y^{2}+z^{2}=1$ cannot be complemented to a matrix-valued field of dimension $3 \times 2$ with rank 2 , because the projection of the complementing field on the tangential bundle of the sphere would produce a nondegenerating tangential field, which is impossible [4].

\section{ACKNOWLEDGMENT}

The author is grateful to the referee for pointing out the book [13] in which rings with condition $(\mathrm{C})$ were examined under the name Hermite algebras.

\section{REFERENCES}

1. G. R. Allan, One-sided inverses in Banach algebras of holomorphic vector-valued functions, J. London Math. Soc. (2) 42 (1967), 463-470.

2. F. Forelli, Bounded holomorphic functions and projections, Illinois J. Math. 10 (1966), 367380. 
3. H. Grauert, Analytische Faserungen über Holomorph Vollständigen Räumen, Math. Ann. 135 (1958), 263-278.

4. D. Husemoller, Fibre bundles, McGraw-Hill, New York, 1966.

5. V. Lin, Holomorphic bundles and multivalued functions from Banach algebra elements, J. Funct. Anal. Appl. 7 (1973), 43-51. (Russian)

6. R. Mortini, The Chang-Marshall algebras, Mitt. Math. Sem. Giessen 185 (1986), 1-76.

7. N. K. Nikolskii, Treatise on the shift operator, Springer-Verlag, Berlin, 1986.

8. M. E. Novodvorskii, On some homotopic invariants of the maximal ideals space, Math. Notes 1 (1967), 487-494. (Russian)

9. J. L. Taylor, Topological invariants of the maximal ideal space of a Banach algebra, Adv. in Math. 19 (1976), 149-206.

10. V. A. Tolokonnikov, Estimates for Carleson Corona theorem, ideals of algebra $H^{\infty}$, Sekefalvi-Nagy problem, Zap. Nauch. Sem. Leningrad. Otdel. Mat. Inst. Steklov. (LOMI) 113 (1981), 178-190. (Russian)

11. _ Algebras of bounded functions with smooth coanalitical part, J. Funct. Anal. Appl. 23 (1989), 88-89. (Russian)

12. __ Generalized Douglas algebras, J. Algebra Anal. 3 (1991), 231-252. (Russian)

13. T. Y. Lam, Serre's conjecture, Lecture Notes in Math., vol. 635, Springer-Verlag, Berlin and New York, 1978, pp. 1-227.

Torgkovskaya str., 2-1, AP. 76, St. Petersburg, Russia 197342 